\title{
ADAPTIVE OPTICS IMAGING OF THE AU MICROSCOPII CIRCUMSTELLAR DISK: EVIDENCE FOR DYNAMICAL EVOLUTION
}

\author{
Stanimir A. Metchev, Joshua A. Eisner, and Lynne A. Hillenbrand \\ Division of Physics, Mathematics, and Astronomy, California Institute of Technology, MC 105-24, Pasadena, CA 91125; \\ metchev@astro.caltech.edu, jae@astro.caltech.edu, lah@astro.caltech.edu \\ AND \\ SEbastian Wolf \\ Max-Planck-Institut für Astronomie, Königstuhl 17, D-69117 Heidelberg, Germany; swolf@mpia-hd.mpg.de \\ Received 2004 September 22; accepted 2004 December 6
}

\begin{abstract}
We present an $H$-band image of the light scattered from circumstellar dust around the nearby ( $10 \mathrm{pc})$ young $\mathrm{M}$ star AU Microscopii (AU Mic, GJ 803, HD 197481), obtained with the Keck adaptive optics system. We resolve the disk both vertically and radially, tracing it over 17-60 AU from the star. Our high angular resolution (0.04 or $0.4 \mathrm{AU}$ per resolution element) observations thus offer the opportunity to probe the morphology of the AU Mic debris disk on solar system scales. We identify substructures (dust clumps and gaps) in the disk that may point to the existence of orbiting planets. No planets are seen in our $H$-band image down to a limiting mass of $1 M_{\mathrm{J}}$ at $>20 \mathrm{AU}$, although the existence of smaller planets cannot be excluded from the current data. Modeling of the disk surface brightness distribution at $H$ band and $R$ band, and of the optical to submillimeter spectral energy distribution, allows us to constrain the disk geometry and the dust grain properties. We confirm the nearly edge-on orientation of the disk inferred from previous observations and deduce that the disk may be clear inward of 1-10 AU. We find evidence for a lack of small grains at $<50 \mathrm{AU}$, either as a result of grain growth or because of destruction by Poynting-Robertson and/or corpuscular drag. A change in the power-law index of the surface brightness profile is observed near $33 \mathrm{AU}$, similar to a feature known in the profile of the $\beta$ Pic circumstellar debris disk. By comparing the timescales for interparticle collisions and Poynting-Robertson drag between the two systems, we argue that the breaks are linked to one or both of these processes.
\end{abstract}

Subject headings: circumstellar matter — instrumentation: adaptive optics -

planetary systems: protoplanetary disks — stars: individual (AU Microscopii) stars: low-mass, brown dwarfs

\section{INTRODUCTION}

The existence of dust disks around main-sequence stars has been known since the first days of the Infrared Astronomy Satellite (IRAS) mission, when Aumann et al. (1984) reported the detection of strong far-infrared (far-IR) excess emission toward Vega ( $\alpha$ Lyr). Over 200 other main-sequence stars have since been reported to possess such excesses, found almost exclusively with IRAS and the Infrared Space Observatory (e.g., Backman \& Paresce 1993; Mannings \& Barlow 1998; Silverstone 2000; Habing et al. 2001; Spangler et al. 2001; Laureijs et al. 2002; Decin et al. 2003), although recently also with Spitzer (e.g., Meyer et al. 2004; Gorlova et al. 2004) and through ground-based submillimeter observations (Carpenter et al. 2005). Too old to possess remnant primordial dust, which would be cleared by radiation pressure and PoyntingRobertson (P-R) drag within several million years (Myr) in the absence of gas, these stars owe their far-IR excess to emission by "debris disks," formed by the collisional fragmentation of larger bodies (the so-called Vega phenomenon; Backman \& Paresce 1993 and references therein). Subsequent imaging at optical to millimeter wavelengths of the nearest subsample of Vega-like stars has resolved intricate disklike structures, with gaps and concentrations (Holland et al. 1998, 2003; Greaves et al. 1998; Schneider et al. 1999; Krist et al. 2000; Koerner et al. 2001; Wilner et al. 2002; Weinberger et al. 2002; Clampin et al. 2003).
The most favored explanation for such structures is the gravitational perturbation by embedded planets orbiting at semimajor axes comparable to the disk size (Moro-Martín \& Malhotra 2002; Kenyon \& Bromley 2004). The existence of perturbing planets may be revealed by clumps of dust trapped in mean motion resonances, as has been suggested for Vega (Wilner et al. 2002; Wyatt 2003), $\epsilon$ Eri (Ozernoy et al. 2000; Quillen \& Thorndike 2002), and Fomalhaut ( $\alpha$ PsA; Wyatt \& Dent 2002; Holland et al. 2003) and observed by the Cosmic Background Explorer along the Earth's orbit (Reach et al. 1995). Stochastic collisions between large planetesimals that result in dust clumps lasting several hundreds of orbital periods are another means of producing disk asymmetries (Stern 1996). Spiral density waves (as seen in the disk of HD 141569A and inferred around $\beta$ Pic; Clampin et al. 2003; Kalas et al. 2000) and warps in the disk inclination (as in the disk of $\beta$ Pic; Heap et al. 2000; Wahhaj et al. 2003) may indicate perturbation by nearby stars (Kalas et al. 2000, 2001; Kenyon \& Bromley 2002; Augereau \& Papaloizou 2004). Finally, dust migration in a gas-rich disk can produce azimuthally symmetric structures, as observed in the HR 4796A circumstellar disk (Takeuchi \& Artymowicz 2001).

High-resolution imaging observations, such as those of HR 4796A (Schneider et al. 1999), $\beta$ Pic (Heap et al. 2000), HD 141569A (Weinberger et al. 1999), and TW Hya (Krist et al. 2000; Weinberger et al. 2002) with the Hubble Space Telescope (HST) can help single out the most likely physical 

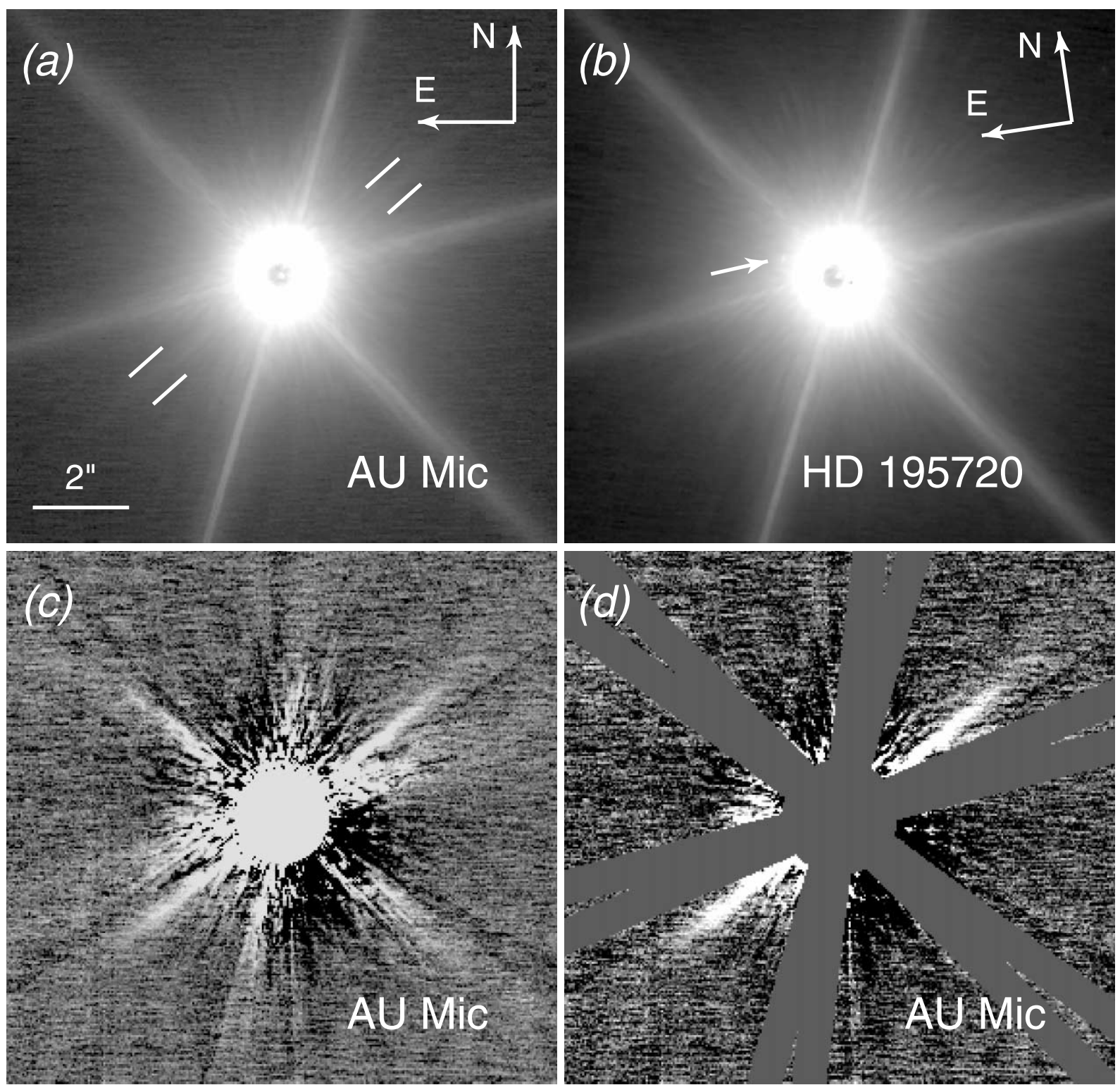

FIG. 1.- $H$-band images of AU Mic. (a) A median-combined image of three sky-subtracted $54.3 \mathrm{~s}$ exposures with the 0.6 coronagraph. The star is visible through the semitransparent coronagraph. The disk is discernible as a faint pair of diametrically opposite spikes along the southeast-northwest direction (traced by the two pairs of parallel lines). (b) An image of the PSF star, HD 195720, with the same coronagraph, scaled to the intensity of the AU Mic image in panel (a). An arrow points to the location of a faint projected companion (§ 3.3). (c) The final median-combined image of AU Mic obtained from PSF-subtracted images with 0"6, 1".0, and 2".0 spot diameters. All surface brightness photometry is performed on this image. $(d)$. Same as in $(c)$, but with an overlaid digital mask covering the star and the telescope diffraction pattern. The residual noise in the central region (3."4 diameter circle) is greater than the surface brightness of the edge-on disk.

process behind the disk morphology. The resolution achievable with adaptive optics (AO) in the near-infrared on large groundbased telescopes rivals that of HST and is the method employed in this paper for investigating disk structure.

The young (8-20 Myr; Barrado y Navascués et al. 1999; Zuckerman et al. 2001) M1 V (Keenan 1983) star AU Mic has a known $60 \mu \mathrm{m}$ excess from IRAS, likely due to orbiting dust (Song et al. 2002). Because of its relative proximity (Hipparcos distance of $9.94 \pm 0.13 \mathrm{pc}$; Perryman et al. 1997), AU Mic is a good target for high-resolution imaging of scattered light to characterize the circumstellar disk morphology. Recent 450 and $850 \mu \mathrm{m}$ observations by Liu et al. (2004) confirmed the existence of dust, and follow-up $R$-band $(0.65 \mu \mathrm{m})$ coronagraphic imaging revealed a nearly edge-on disk extending $210 \mathrm{AU}$ (Kalas et al. 2004) from the star. Because the age of AU Mic is greater than the collision timescale between particles in the disk
(0.5-5 Myr at $200 \mathrm{AU})$, Kalas et al. (2004) infer that most of the dust particles have undergone at least one (destructive) collision, and hence the AU Mic disk is a debris disk. However, because the P-R timescale for $0.1-10 \mu \mathrm{m}$ particles at $\gtrsim 100 \mathrm{AU}$ from the star is greater than the stellar age, Kalas et al. expect that most of the disk at these radii consists of primordial material. From the lack of excess at $25 \mu \mathrm{m}$, Liu et al. infer an innerdisk edge at 17 AU from the star, or 1".7 at the distance of AU Mic. They speculate that such a gap may have been opened by an orbiting planet that, given the youth of the system, could be detectable in deep AO observations in the near-IR. Such high-contrast observations could also be used to search for morphological signatures of planet/disk interaction.

The AU Mic circumstellar disk is not resolved with the $14^{\prime \prime}$ beam of the James Clerk Maxwell Telescope Submillimeter Common-User Bolometric Array (JCMT/SCUBA) observations 
of Liu et al. (2004), and the seeing-limited optical coronagraphic observations of Kalas et al. (2004) are insensitive to the disk at separations $<5^{\prime \prime}$. Taking advantage of the higher angular resolution and dynamic range achievable with $\mathrm{AO}$ on large telescopes, Liu (2004) used the Keck AO system to investigate the disk morphology at separations as small as 15-20 AU from the star. We present our own set of Keck AO data, which confirms Liu's observations and places upper limits on the presence of potential planetary companions. In addition, we combine our spatially resolved $H$-band information with the $R$-band imaging data from Kalas et al. (2004), and with the optical to submillimeter spectral energy distribution (SED) of AU Mic from Liu et al. (2004), to put self-consistent constraints on the disk morphology and the dust properties, as done previously for $\beta$ Pic (Artymowicz et al. 1989). We use a full three-dimensional radiative transfer code to model simultaneously the SED and the $H$-band and $R$-band surface brightness profiles (SBPs). We find that a model consisting of a single dust population does not reproduce the observed break in the $H$-band SBP, whereas a two-component dust model, as proposed for $\beta$ Pic, fits the data well. Drawing from a comparison with the $\beta$ Pic system, we deduce that dynamical evolution of the disk provides the simplest explanation for the morphology of the SBPs of both disks.

\section{OBSERVATIONS AND DATA REDUCTION}

We observed AU Mic at $H$ band $(1.63 \mu \mathrm{m})$ with the NIRC2 instrument (K. Matthews et al. 2005, in preparation) and the AO system (Wizinowich et al. 2000) on the Keck II telescope. The data were acquired on 2004 June 5 under photometric conditions. We employed coronagraphic spots of different sizes $(0$ "'6-2".0 diameter) to block out the light from the star. The observations were taken with the wide, $0^{\prime \prime} 04$ pixel $^{-1}$ camera in NIRC2, which delivers a $41^{\prime \prime} \times 41^{\prime \prime}$ field of view on the $1024 \times$ 1024 InSb ALADDIN III array.

We obtained nine $54 \mathrm{~s}$ exposures at $H$ band, three with each of the $0.6,11^{\prime \prime} 0$, and $2^{\prime \prime} 0$ diameter coronagraphic spots. We observed the nearby ( ${ }^{\circ} .4$ separation) M2/3 III star HD 195720 with similar colors, but 0.9 mag brighter than $\mathrm{AU}$ Mic at $H$ band, as a point-spread function (PSF) standard. We spent equal amounts of time on the target, on the PSF star, and on the sky. The observations were carried out according to the following sequence, repeated three times: three exposures of AU Mic, three exposures of HD 195720, and three exposures of the blank sky (taken at three dithered positions: $60^{\prime \prime}, 50^{\prime \prime}$, and $40^{\prime \prime}$ away from the PSF star). The total on-source exposure time was 8.1 minutes. Throughout the observations, the field rotator was set in "position angle mode," preserving the orientation of the sky on the detector. The image quality was estimated from the Strehl ratios of point sources observed at higher spatial resolution (with the narrow camera, 0.01 pixel $^{-1}$ ) at the beginning of each night. Our $H$-band images had Strehls of $17 \%-20 \%$.

Data reduction followed the standard steps of sky subtraction, flat fielding, and bad pixel correction. The images in the individual sets of three exposures were then median combined to improve the sensitivity to faint objects. The AU Mic dust disk was barely discernible at this stage (Fig. 1a). To enhance the visibility of the dust disk, we subtracted the stellar PSF. The PSF was obtained by first rotating the image of HD 195720 to match the orientation of the diffraction pattern in the AU Mic image, and then by scaling it by a centrally symmetric function $f(r)$ to match the stellar profile of AU Mic. The objective of the scaling was to compensate for variations in the seeing halo between AU Mic and the control star caused by changing at-

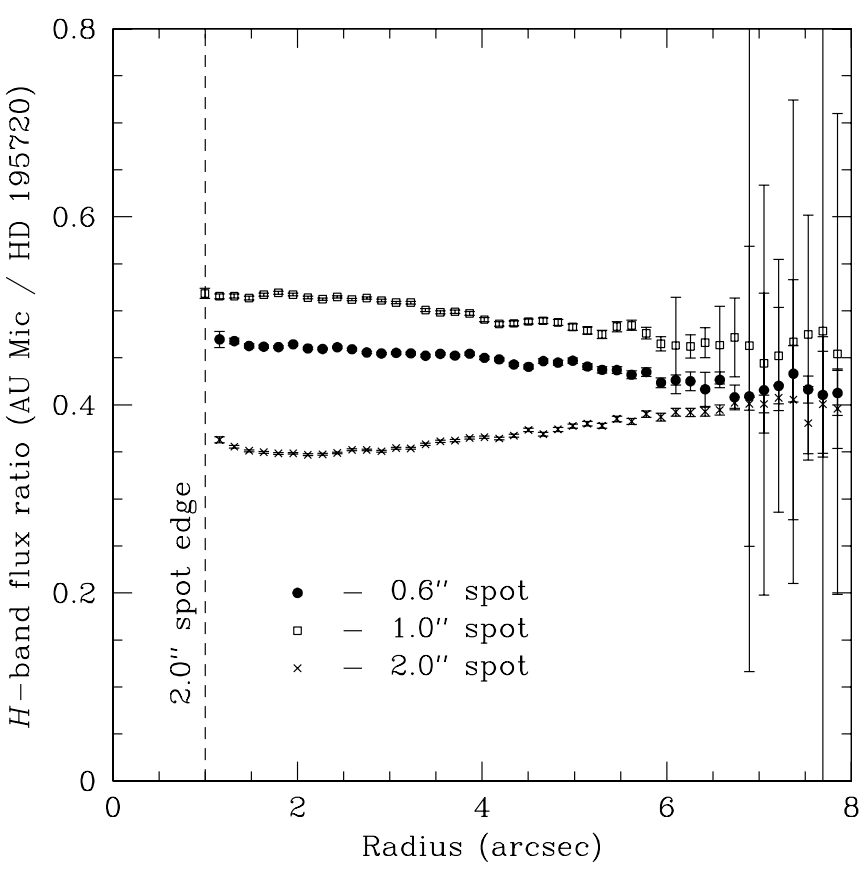

FIG. 2.-Median ratios $f(r)$ of the radial profiles of AU Mic and HD 195720 (the PSF star) at $H$ band for the images taken with the various coronagraphs. The vertical dashed line shows the edge of the largest coronagraph. Our PSF images were multiplied by $f(r)$ before subtracting them from the corresponding AU Mic images. For any given coronagraphic spot size, $f(r)$ varies by less than $15 \%$ in the range $r=1^{\prime \prime}-8^{\prime \prime}$.

mospheric conditions and fluctuating quality of the AO correction. The function $f(r)$ was obtained as the median radial profile of the ratio of the AU Mic to the HD 195720 images, with the telescope spikes and the edge-on disk masked. Figure 2 shows $f(r)$ for the images with the three different coronagraphic spot sizes. Two remarks on this procedure should be made here. First, the function $f(r)$ does not vary by more than $15 \%$ for any given spot size, and for large radii tends to 0.4 , the $H$-band flux ratio between AU Mic and the PSF star. Second, because the AU Mic disk happens to be edge-on, the centrally symmetric scaling of the PSF does not introduce any spurious features in the result and so does not interfere with the morphology of the disk. The procedure would not be viable for disks that are not viewed edge-on.

The scaled version of HD 195720 for the image with the 0 ".6 coronagraph is presented in Figure $1 b$. Figure $1 c$ shows the final AU Mic image, obtained by median combining all coronagraphic exposures. In Figure $1 d$ a digital mask has been employed to enhance the appearance of the circumstellar disk. The mask encompasses the innermost 1"7 from AU Mic, as well as the hexagonal diffraction spikes from two of the three image sequences in which they did not subtract well (as seen in Fig. 1c).

For flux calibration we adopted the 2MASS magnitude for AU Mic $(H=4.831 \pm 0.016)$ and relied on residual transmission through the NIRC2 coronagraphic spots. The PSF star, HD 195720, is saturated in 2MASS and therefore unusable for flux calibration. We measured the flux from AU Mic through the $1{ }^{\prime \prime} 0$ and 2.0 coronagraphs in a 6 pixel $(0.24)$ diameter aperture. From noncoronagraphic images taken with the 10 mas $\mathrm{pixel}^{-1}$ camera on NIRC2, we found that this aperture contained $68 \%$ of the total power in the PSF. The $H$-band transmissivity of the $2^{\prime \prime}$ NIRC2 coronagraph was thus measured at $(7.32 \pm 0.24) \times$ $10^{-4}$ (extinction of $7.84 \pm 0.03 \mathrm{mag}$ ). 
AU Mic is known to exhibit a large $V$-band photometric amplitude $(0.35 \mathrm{mag})$ due to star spots with a period of 4.9 days (Torres \& Ferraz Mello 1973). Although we do not expect measurable variability over the $\approx 1 \mathrm{hr}$ time span of our observations, our absolute flux calibration is uncertain. Nevertheless, in the near-IR the contrast between the spots and the stellar photosphere is less pronounced than in the optical, so the uncertainty is also smaller, likely of order $\lesssim 0.1 \mathrm{mag}$.

The absolute orientation of the dust disk arms was calibrated through observations of a binary star standard, WDS 18055+ 0230, with well-known orbital elements (grade 1; Hartkopf \& Mason 2003; Pourbaix 2000). The $y$-axis of the NIRC2 detector was measured to be offset by $1.24 \pm 0.10$ clockwise from north. All position angles quoted below have been corrected for this offset.

\section{RESULTS AND ANALYSIS}

\subsection{Circumstellar Dust Morphology}

The disk is seen out to a distance of $\sim 6^{\prime \prime}$ ( $60 \mathrm{AU}$ ) from the star in our combined $H$-band image (Figs. $1 c-1 d$ ). It can be traced inward to $\approx 1.77$ (17 AU) from the star, at which point residual speckle noise from the PSF subtraction overwhelms the emission from the disk. Thus, our imaging data cannot directly test the existence of the proposed disk clearing interior to $17 \mathrm{AU}$ from the star (Liu et al. 2004). We confirm the sharp midplane morphology of the disk (Kalas et al. 2004; Liu 2004), indicating a nearly edge-on orientation, and resolve the disk thickness, with the southeast arm appearing somewhat thicker (FWHM = 2.8-4.4 AU) than the northwest arm (FWHM $=2.2-4.0 \mathrm{AU})$. There is also evidence of an increase in the FWHM of each of the arms with separation: from 2.2-2.8 AU at $20 \mathrm{AU}$ to 4.0 4.4 $\mathrm{AU}$ at $40 \mathrm{AU}$ from the star, indicating a potential nonzero opening angle of the disk. Within $5^{\prime \prime}$ of AU Mic, the position angles (P.A.s) of the two sides of the disk are nearly $180^{\circ}$ away from each other: we measure P.A. $=310^{\circ} .1 \pm 0.2$ for the northwest arm and P.A. $=129^{\circ} .5 \pm 0.4$ for the southeast arm. These P.A.s are in agreement with those reported in $311^{\circ} .4 \pm 1^{\circ} .0$ and $129^{\circ} 3 \pm 0.8$ Liu (2004), although more accurate, likely as a result of our proper calibration of the orientation of the NIRC2 detector $(\S 2)$.

The radial SBP of the disk was measured on the reduced image (Fig. 1c; before applying the digital mask) using the IRAF task POLYPHOT. The photometry regions are indicated by the rectangles overlapped onto a contour map of the image in Figure $3 a$. We used 4 pixel $\times 12$ pixel $\left(00^{\prime \prime} 16 \times 00^{\prime \prime} 48\right)$ rectangular apertures, where the long side of the rectangular regions was chosen to span 1-2 FWHMs of the disk thickness and was aligned normally to the disk arm. The distance between the aperture centers was 4 pixels $\left(0{ }^{\prime \prime} 16\right)$. Even though the image was PSF- and sky-subtracted, to offset for flux biases introduced by the centrally symmetric scaling of the PSF $(\S 2)$ we employed additional background subtraction, with the background flux estimated as the median pixel value in 0.16 wide concentric annuli centered on the star. The photometric uncertainty was estimated as the quadrature sum of the standard deviation of the background plus the photon noise from the disk. For the standard deviation of the background we adopted the $\mathrm{rms}$ of the pixel values in the annulus, multiplied by $(\pi / 2)^{1 / 2}$ (to properly account for the standard deviation of the median; Kendall \& Stuart 1977) and normalized by the size of the photometry aperture.

The radial SBPs of the northwest (upward pointing triangles) and southeast (downward pointing triangles) arms of the pro-

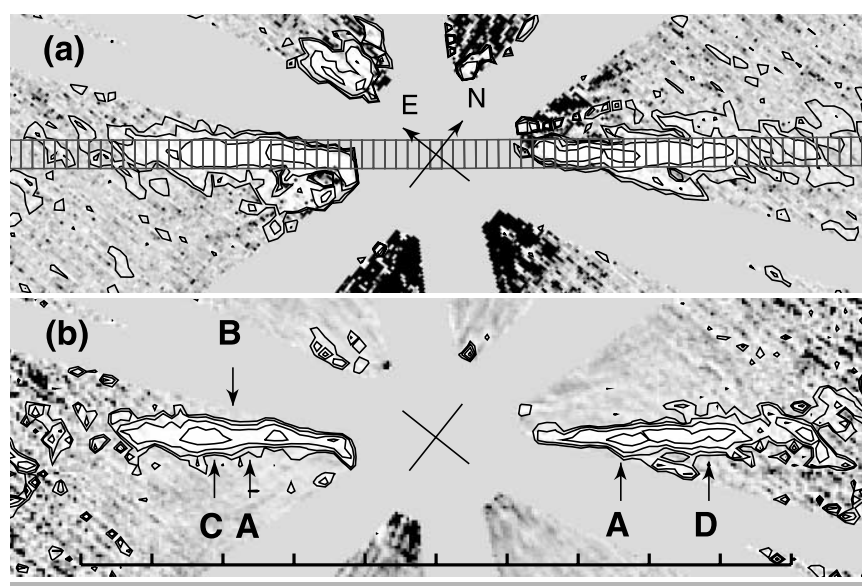

(c)

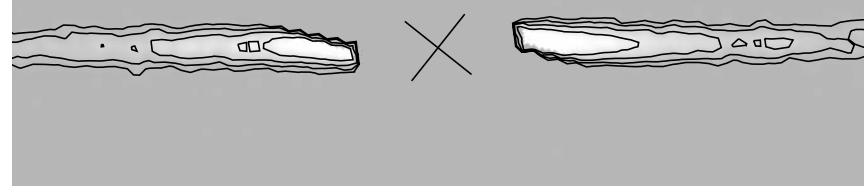

FIG. 3.- Image slices $\left(12 . " 0 \times 4{ }^{\prime \prime} 0\right)$ along the AU Mic disk, with the southeast arm oriented horizontally. (a) Locations of the photometry regions for measuring the disk surface brightness superposed on the final masked image of AU Mic. The regions are 0 " $16 \times 0$ ". 48 and sample $1-2$ FWHMs of the disk thickness. The circular mask is $2^{\prime \prime}$ in radius. The crossed arrows mark the location of AU Mic. The contour levels trace surface brightness from 17.5 to $14.3 \mathrm{mag}$ $\operatorname{arcsec}^{-2}$ in steps of $0.8 \mathrm{mag} \operatorname{arcsec}^{-2}$. (b) Small-scale structure in the AU Mic disk. The capital letters correspond to substructures identified by Liu (2004). The bar at the bottom is $10^{\prime \prime}$ (100 AU) long, extending from -50 to $+50 \mathrm{AU}$ along the disk plane, with tick marks every $10 \mathrm{AU}$. To enhance the appearance of the clumps in the disk, we have multiplied the pixel values by the square of the distance from the star. The contour levels follow a squared intensity scale. (c) The preferred scattered-light model of the AU Mic disk at $H$ band, created using the MC3D code ( $\S 4$; Table 1$)$. The same software mask used in the other two panels has been applied. The contour levels follow the same spacing as in panel $(a)$. No background noise is added, although Poisson noise "clumps" due to the low signal-to-noise ratio of the model can be seen. These do not represent discrete physical structures.

jected disk are shown in Figure 4. The two SBPs agree well throughout the region over which we can trace the disk (17$60 \mathrm{AU})$. Unlike the $R$-band SBP over $50-210 \mathrm{AU}$, the $H$-band SBP over 17-60 AU from the star cannot be fitted by a single power law. Instead, the SBPs of both arms "kink" and flatten inward of 30-40 AU, with the transition being more abrupt in the southeast arm at $\approx 33 \mathrm{AU}$ and more gradual in the northwest arm. While the power-law exponent of the mean SBP over the entire range ( $17-60 \mathrm{AU})$ is $-2.3 \pm 0.2$, over $17-33 \mathrm{AU}$ separations it flattens to $-1.2 \pm 0.3$, while over 33-60 AU it increases to $-4.0 \pm 0.6$. These are consistent with the measurements of Liu (2004) over the same separation ranges.

A closer look at the SBP of the northwest and southeast arms reveals several small-scale asymmetries, all of which can be linked to regions of nonuniform brightness in the AU Mic disk (Fig. $3 a$ ). The substructure is enhanced by scaling the reduced image by a radially symmetric function centered on the star, with magnitude proportional to the radius squared (Fig. $3 b$ ). The lettered structures denote features identified in the (deeper) image of Liu (2004): clumps of enhanced emission (A and C), a gap (B), and a region elevated with respect to the inner-disk midplane (D). In general, we confirm the presence of these features in the AU Mic disk, although the gap (B) and the clump (A) appear misplaced by 2-3 AU toward the star in our image. The 


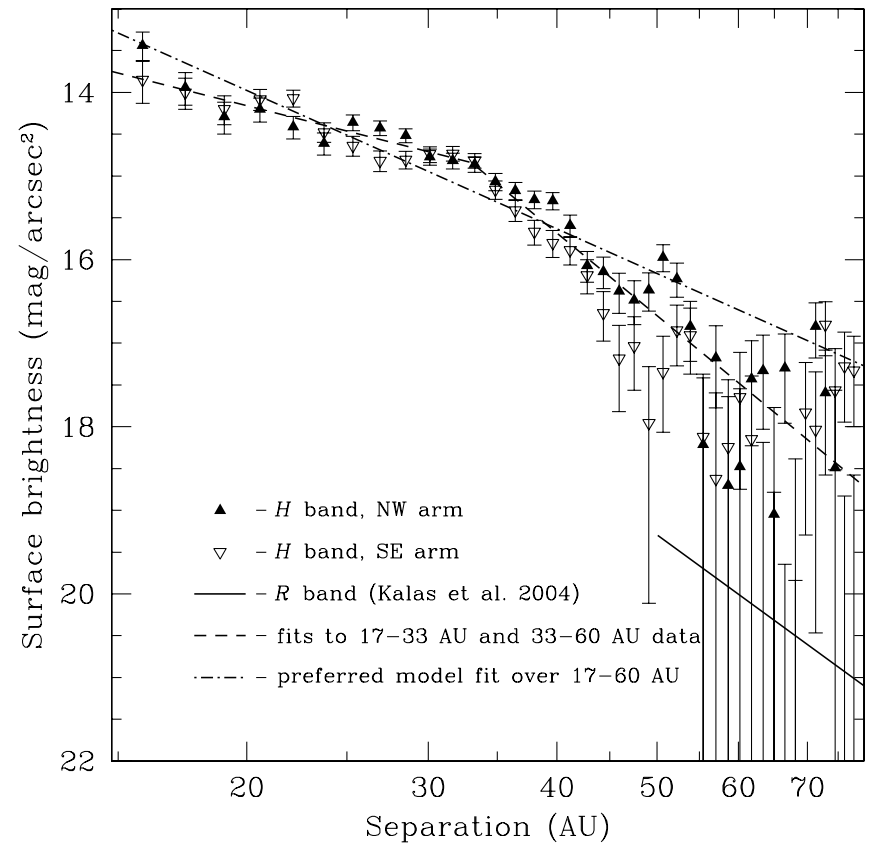

FIG. 4. $-H$-band surface brightness profiles of the northwest (upward pointing filled triangles) and the southeast (downward pointing open triangles) arms of the AU Mic disk. A gradual flattening of the SBPs of both arms is observed inward of 30-40 AU. The dashed lines represent the power-law fits to the mean SBP over 17-33 AU (index of $-1.2 \pm 0.2$ ) and 33-60 AU (index of $-4.0 \pm 0.6)$. The solid line represents the mean $R$-band SBP from Kalas et al. (2004) with a power-law index of -3.75 . The dot-dashed line shows our preferred model with a power-law index -2.2 , matching that $(-2.3 \pm 0.2)$ of the mean SBP over 17-60 AU.

northwest arm also looks more uniform in brightness between 17 and $40 \mathrm{AU}$ in our image compared to that in Figures 3 and 4 of Liu (2004), where clump A is very prominent. These discrepancies may be caused by residual speckle noise from the PSF subtraction in either data set. In addition to the features described by Liu, we see a faint clump in the northwest arm at $\gtrsim 50 \mathrm{AU}$ from the star, coincident with the location of the bump in the SBP of this arm (Fig. 4). The concentration is not reported by Liu, and being at a relatively low signal-to-noise ratio $(\approx 3)$ may be a noise spike.

\subsection{Disk Luminosity, Optical Depth, and Geometry}

The integrated disk brightness (over 17-60 AU from the star) is $2.7 \pm 0.8 \mathrm{mJy}$ at $H$ band; hence, $L_{\text {scat }} / L_{*}=2.3 \times 10^{-4}$. This is comparable to the fractional dust luminosity in emitted midIR to submillimeter light, $f_{d}=L_{\mathrm{IR}} / L_{*}=6 \times 10^{-4}$ (Liu et al. 2004), and hence suggests that the disk midplane may be optically thin to radiation at wavelengths as short as $1.6 \mu \mathrm{m}$, near the peak of the AU Mic spectrum. Indeed, $f_{d}$ is similar to that of other known debris disks $\left(10^{-5}\right.$ to $10^{-3}$; e.g., Sylvester \& Mannings 2000; Habing et al. 2001; Spangler et al. 2001), all of which are optically thin to ultraviolet and optical light in the direction perpendicular to the disk plane $\left(\tau_{\perp} \ll 1\right)$. In the midplane, the optical depth of grains along a radial line from the star to infinity is $\tau_{\|} \sim f_{d} / \sin \delta$ if the grains are in a "wedge" or "flaring" disk with thickness proportional to radius and opening angle $2 \delta$ (Backman \& Paresce 1993). Because of the generally unknown viewing geometry of circumstellar disks, $\tau_{\|}$ tends to be poorly constrained.

We can estimate the maximum allowed opening angle $2 \delta$ from the observed disk thickness. However, $\delta$ is degenerate with the disk inclination $i$. For smaller values of $i, \delta$ will be smaller because of projection effects. Assuming a perfectly

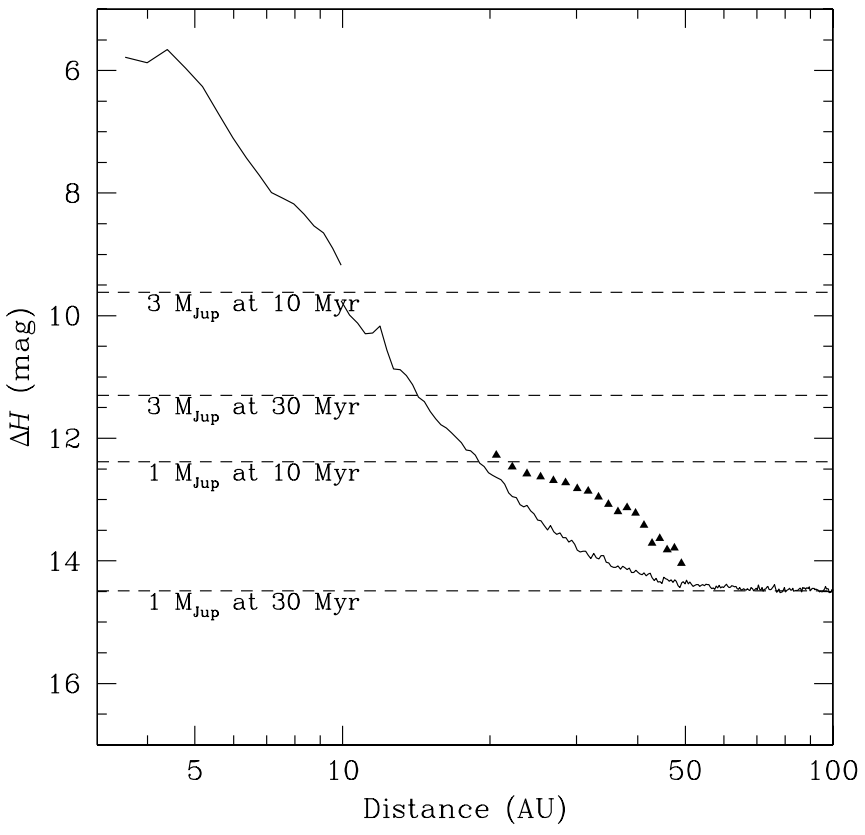

FIG. 5.- $H$-band $5 \sigma$ detection limits for companions to AU Mic. The solid line delineates the limits in regions away from the disk. The break at $10 \mathrm{AU}\left(1^{\prime \prime}\right)$ corresponds to the edge of the largest $\left(2^{\prime \prime}\right.$ diameter) coronagraphic spot. To determine the detection limits at separations $\leq 1^{\prime \prime}$, we used only the series of images taken with the 0 ." 6 spot, constituting a third of the total exposure time. Thus, our sensitivity at small separations is somewhat worse than what the extrapolation from distances $>10$ AU would predict. The triangles trace the poorer sensitivity to point sources in the plane of the disk. Limited experimentation with planting artificial sources in the image confirmed these detection limits. The dashed lines indicate the expected contrast for $1 M_{\mathrm{J}}$ and $3 M_{\mathrm{J}}$ planets around AU Mic (Burrows et al. 1997) for system ages of 10 and $30 \mathrm{Myr}$.

flat, thin disk, we find a lower limit on the inclination $i>87^{\circ}$ over 20-50 AU. Because the projected appearance of an inclined disk of zero thickness is inconsistent with the apparent thickening of the disk with increasing separation $(\S 3.1)$, we infer that the disk likely has nonzero scale height and/or opening angle and is viewed within only a degree of edge-on. To put an upper limit on $\delta$, we observe that at a radius of $40 \mathrm{AU}$ the disk FWHM is $\sim 4$ AU. Thus, we obtain that for $i \lesssim 90^{\circ}$, $\delta \lesssim 3^{\circ}$. Hence, $\tau_{\|} \geq 6 \times 10^{-3}$, and probably less than unity; i.e., the disk is optically thin in the radial direction.

\subsection{Detection Limits on Substellar Companions}

Dynamical influence by embedded planets is a frequently invoked explanation for substructure in dust disks. Because of its youth, proximity, and late spectral type, AU Mic is an ideal target for direct imaging of planets. However, no point sources are seen in our combined 8.1 minute PSF-subtracted $H$-band exposure. Figure 5 delineates our $5 \sigma$ sensitivity limits as a function of angular separation from the star in the PSF-subtracted image. The $1 \sigma$ level at each distance is defined as the rms scatter of the pixel values in 1 pixel wide annuli centered on the star. This was divided by $(28.3-1)^{1 / 2}$ to adjust for the finite size of the aperture used for photometry ( 6 pixel diameter, or an area of 28.3 pixel $^{2}$ ). The sensitivity to point sources along the disk is up to 1 mag poorer because of the higher photon noise. This is shown in Figure 5 with the triangles each of which has the photon noise from the disk signal added in quadrature. These detection limits, calculated in a statistical manner, were confirmed through limited experiments with artificially planted stars.

At the location of the inferred gap in the southeast arm $(\sim 25-$ $30 \mathrm{AU})$, we can detect planets down to 1 Jupiter mass $\left(M_{\mathrm{J}}\right)$ 
for an assumed stellar age of 10 Myr and using brown dwarf cooling models from Burrows et al. (1997). Dynamical models of planet-disk interactions in other systems exhibiting similar disk morphology ( $\epsilon$ Eri and $\alpha$ Lyr) require planets $0.1 M_{\mathrm{J}}-3 M_{\mathrm{J}}$ (Quillen \& Thorndike 2002; Wilner et al. 2002). Provided that the clumps in the AU Mic disk are caused by such a planet, our point-source detection limits constrain its mass to the lower part of this range.

A faint candidate companion is seen around our PSF star, HD $195720(H=3.88 \pm 0.24$ from 2MASS $)$. The object is $9.5 \pm 0.2 \mathrm{mag}$ fainter at $H$, at a projected separation of 1.19 and P.A. of $81^{\circ}$ (Fig. 1b). Given that HD 195720 is a distant giant star (spectral type M2-3 III from SIMBAD), if associated, the companion would be a main-sequence $\mathrm{K}$ star. Because of the large magnitude difference, the presence of the projected companion does not affect our PSF subtraction or the analysis of the AU Mic circumstellar disk.

\section{DUST DISK MODELING}

It has already been suggested that the structures in the AU Mic disk (dust concentrations, gaps, and vertically displaced clumps) are likely signposts of the existence of perturbing planetary mass bodies in the AU Mic disk (Liu 2004). The proposed clearing in the disk inward of 17 AU (Liu et al. 2004) supports such a hypothesis. From our imaging data we cannot trace the disk to separations $<17$ AU to directly test the existence of a gap. However, by combining our knowledge of the optical to submillimeter data on the AU Mic debris disk with an appropriate model, we can still probe some of the physical properties of the disk, including the size of the inner gap. In this section we present results from a three-dimensional continuum radiative transfer code, MC3D (Wolf \& Henning 2000; Wolf 2003), to simultaneously model the AU Mic SED and the scattered light from the disk and to place constraints on the dust grain size distribution, the radial particle density distribution, and the inner-disk radius.

\subsection{Model and Method}

The MC3D code is based on the Monte Carlo method and solves the radiative transfer problem self-consistently. It estimates the spatial temperature distribution of circumstellar dust and takes into account absorption and multiple scattering events. Given the nonvanishing midplane optical depth of the AU Mic disk ( $\S 3.2$ ), we believe that the use of a multiscattering approach is warranted. The code employs the concept of enforced scattering (Cashwell \& Everett 1959), where in a medium of optical depth $\tau$, a fraction $e^{-\tau}$ of each photon leaves the model space without interaction, while the remaining part $\left(1-e^{-\tau}\right)$ is scattered. The code is therefore applicable to the low-density environments of circumstellar debris disks. The dust grains are assumed to be spherical with a power-law size distribution $n(a) \propto a^{-3.5}$ (Mathis et al. 1977). We used a standard interstellar medium (ISM) mixture of $62.5 \%$ astronomical silicate and $25 \%+12.5 \%$ graphite (Draine \& Malhotra 1993; Weingartner \& Draine 2001), with optical properties from Draine \& Lee (1984). The extinction and scattering cross sections and the scattering distribution function are modeled following the Mie scattering algorithm of Bohren \& Huffman (1983).

We use the MC3D code to model the $R$ - and $H$-band scattered light in the AU Mic disk and the IR to submillimeter excess in the SED. The AU Mic photosphere is best approximated by a 3600 K NextGen model (Hauschildt et al. 1999), as expected from its spectral type (M1 V). The fit was performed over the $1-12 \mu \mathrm{m}$ wavelength range, where the emission is photospheric. Data from the literature at shorter wavelengths were ignored, as they are not taken simultaneously, and hence are strongly affected by the $\Delta V=0.35$ mag variability of the star. By matching the model $K_{S}$-band flux density to that of a blackbody of the same temperature and adopting the Hipparcos distance of $9.94 \mathrm{pc}$ to the star, we find that its luminosity and radius are $0.13 L_{\odot}$ and $0.93 R_{\odot}$, respectively. For the debris disk we adopt a flat (unflared) geometry with a number density profile proportional to $r^{-\gamma}$, where $r$ denotes radial distance from the star and $\gamma$ is a constant. We set the outer radius of the model to $1000 \mathrm{AU}$, so that it is larger than the size of the $R$-band scattered-light emission (210 AU), and of the JCMT/SCUBA beam used for the submillimeter measurements (FWHM of $\left.14^{\prime \prime}=140 \mathrm{AU}\right)$. The disk inclination and opening angle were already constrained in $\S 3.2$. For our modeling purposes we assume $i=89^{\circ}, \delta=0^{\circ}$, and a flat disk model with a constant scale height $h=0.8 \mathrm{AU}$. We find that models based on these parameters approximate the mean observed disk thickness well.

The remaining free parameters in the disk model are the exponent of the volume density profile $\gamma$, the dust mass $M_{\text {dust }}$, the minimum and maximum dust grain sizes $a_{\min }$ and $a_{\max }$, and the inner radius $r_{\text {in }}$. A fit to the mean northwest plus southeast SBP between 17 and 60 AU results in a best-fit power-law index of $-\nu=-2.3 \pm 0.2$, indicating that the number density profile varies as $r^{-1.3 \pm 0.2}$. Here we have assumed $\gamma=\nu-1=1.3 \pm$ 0.2 , as is true for an edge-on disk of isotropically scattering grains (Nakano 1990; Backman et al. 1992). ${ }^{1}$ This value is in agreement with the range inferred for P-R drag dominated disks (1.0-1.3; e.g., Briggs 1962). Given the error on our fit, we decided to fix the value of the power-law index at the theoretically expected value of $\gamma=1.0$ for a continuously replenished dust cloud in equilibrium under P-R drag (Leinert et al. 1983; Backman \& Gillett 1987). The effects of varying $\gamma$ are considered at the end of $\S 4.2$.

\subsection{Breaking Degeneracies in the Model Parameters}

We subsequently follow a trial-and-error by-eye optimization scheme to determine the values of $M_{\text {dust }}, a_{\min }, a_{\max }$, and $r_{\text {in }}$. With a sophisticated dust disk model containing many parameter choices, it is possible to find combinations of parameters that have degenerate effects on the SED and/or on the SBP. By fitting simultaneously the SED and the imaging data we can avoid some, but not all, of the complications. Here we discuss the specific degeneracies and how we can break them via the observational constraints in hand. We first consider the interaction between $M_{\mathrm{dust}}, a_{\mathrm{min}}$, and $a_{\mathrm{max}}$, which are strongly degenerate. We then consider the effect of changing $r_{\text {in }}$, which is more weakly coupled with the rest of the parameters. Finally, we extend our discussion to consider variations in the power-law index $\gamma$, which is otherwise kept fixed during the modeling.

The dust mass $M_{\text {dust }}$ and the minimum and maximum grain sizes, $a_{\min }$ and $a_{\max }$, have degenerate effects on both the SBP and the SED. Decreasing $M_{\text {dust }}$, or increasing $a_{\min }$ or $a_{\max }$, results in a decrease in the amount of mass residing in small grains (the bulk of the scatterers) and lowers the flux of the SBP. Each of these changes similarly lowers the thermal SED flux. However, dust mass variations can be disentangled from grain size

\footnotetext{
${ }^{1}$ Forward scattering, to the extent to which it is characteristic of the dust grains in the AU Mic disk, tends to increase $\gamma$. Even though forward scattering is ignored in the approximation $\gamma=\nu-1$, it is modeled by the MC3D code, where its amount is determined by the input grain parameters and Mie theory.
} 

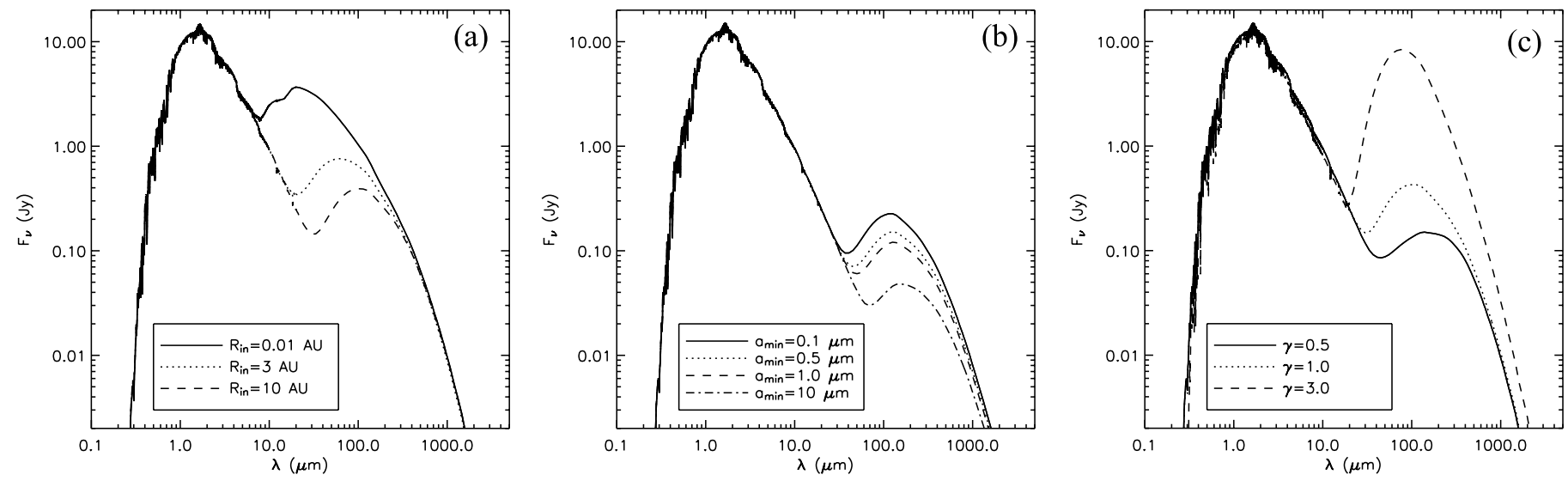

FIG. 6. - Examples of degeneracies in the SED. Larger inner gap radii (a) are degenerate with larger minimum grain sizes $(b)$ and shallower number density distributions $(c)$. Various combinations of these parameters can produce roughly the same SED. The normalizations of the emitted mid-IR flux from the disk are different among the three panels.

variations because of the different magnitudes of their effects on the SBP and on the SED. Optically thin thermal emission is a more accurate proxy of dust mass, whereas optical-IR scattering is more sensitive to small differences in the mean grain size. We therefore constrain $M_{\text {dust }}$ from the submillimeter data, while we use the color and absolute flux of the scattered light to determine $a_{\min }$ and $a_{\max }$. Here we should note that $M_{\text {dust }}$ represents only the dust mass contained in grains comparable or smaller in size than the maximum wavelength $\left(\lambda_{\max }\right)$ at which thermal emission is observed. In the case of AU Mic, the currently existing longest wavelength observations are at $850 \mu \mathrm{m}$ (Liu et al. 2004). Consequently, we are free to adjust $a_{\min }$ and $a_{\max }$, as long as $a_{\min } \leq a_{\max } \lesssim 1 \mathrm{~mm}$. We will not consider cases for which $a_{\max }>1 \mathrm{~mm}$.

As a first step in finding the optimum model parameters, we confirm the Liu et al. (2004) estimate of the dust mass, $M_{\text {dust }}=0.011 M_{\oplus}$, calculated from the $850 \mu \mathrm{m}$ flux. This value matches the submillimeter data points for a wide range (an order of magnitude) of minimum and maximum grain sizes, whereas changing $M_{\text {dust }}$ by a factor of $>1.5$ introduces significant discrepancies from the observed $850 \mu \mathrm{m}$ emission. As a next step, we constrain $a_{\min }$ by modeling the optical near-IR color of the scattered light in the overlap region (50-60 AU) between the
Kalas et al. (2004) $R$-band and our $H$-band data. Because the size of the smallest grains is likely comparable to the central wavelengths of the $R$ and $H$ bands, $R-H$ is a sensitive diagnostic for $a_{\min }$. We smooth the $R$ - and $H$-band model images to the respective image resolutions (1". 1 at $R$ and $0^{\prime \prime} 04$ at $H$ ) and use the appropriate aperture widths ( $\left(1{ }^{\prime \prime} 2\right.$ at $R$, Kalas et al. 2004; 0 ".48 at $H$, this work). For the adopted grain size distribution we find that models with $a_{\min } \approx 0.5 \mu \mathrm{m}$, with a probable range of $0.3-1.0 \mu \mathrm{m}$, best approximate the disk color. Having constrained $M_{\text {dust }}$ and $a_{\min }$, we find that $a_{\max }=300 \mu \mathrm{m}$ matches best the $R$ - and $H$-band flux levels of the disk. The probable range for $a_{\max }$ is $100-1000 \mu \mathrm{m}$. Since we have not considered models with $a_{\max }>1 \mathrm{~mm}$, we cannot put an upper limit to the maximum grain size.

The inner radius $r_{\text {in }}$ of the disk is degenerate with the mean dust grain size in the SED. Greater values of $r_{\text {in }}$ decrease the mid-IR flux and shift the peak of the excess to longer wavelengths (Fig. $6 a$ ), as do greater values of $a_{\min }$ (Fig. $6 b$ ) and $a_{\max }$. Because the AU Mic disk is optically thin in the midplane $(\S 3.2)$, the inner-disk radius has no effect on the flux and color of the SBP, facilitating the isolation of the $r_{\text {in }}$ parameter. Having already determined $a_{\min }$ and $a_{\max }$, we find $r_{\text {in }} \approx 10 \mathrm{AU}$ : smaller than the $17 \mathrm{AU}$ gap estimated from the single-temperature

TABLE 1

Preferred Model Parameters for the AU Mic System

\begin{tabular}{|c|c|c|}
\hline Parameter & Value & Range \\
\hline 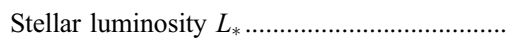 & $0.13 L_{\odot}$ & Fixed \\
\hline 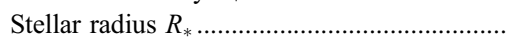 & $0.93 R_{\odot}$ & Fixed \\
\hline 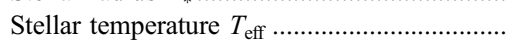 & $3600 \mathrm{~K}$ & Fixed \\
\hline Dust mass $M_{\text {dust }}$ & $0.011 M_{\oplus}$ & $0.008-0.016 M_{\oplus}$ \\
\hline 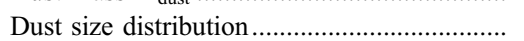 & $a^{-3.5}$ & Fixed \\
\hline 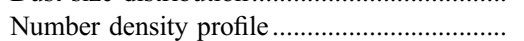 & $r^{-1.0}$ & -0.2 to -3.0, fixed $^{\mathrm{a}}$ \\
\hline 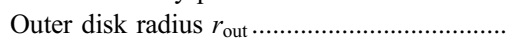 & $1000 \mathrm{AU}$ & Fixed \\
\hline Inner disk radius $r_{\text {in }}$ & $10 \mathrm{AU}$ & $1-10 \mathrm{AU}$ \\
\hline Inclination angle $i$ & $89^{\circ}$ & $\gtrsim 89^{\circ}$ \\
\hline 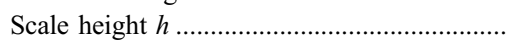 & $0.8 \mathrm{AU}$ at $r=40 \mathrm{AU}$ & $\lesssim 1.0 \mathrm{AU}$ at $r=40 \mathrm{AU}$ \\
\hline 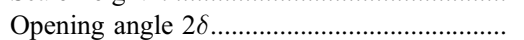 & $0^{\circ}$ & $\lesssim 6^{\circ}$ \\
\hline 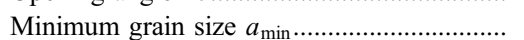 & $0.5 \mu \mathrm{m}$ & $0.3-1 \mu \mathrm{m}$ \\
\hline 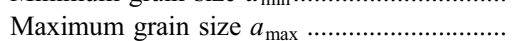 & $300 \mu \mathrm{m}$ & $100-1000 \mu \mathrm{m}^{\mathrm{b}}$ \\
\hline
\end{tabular}

${ }^{a}$ Treated as a fixed parameter during the MC3D model fitting. The listed range corresponds to the range of fits to the SBP over 17-60 AU.

$\mathrm{b}$ The SED data are not sensitive to emission from grains $\gtrsim 1000 \mu \mathrm{m}$ in size, so we have not run models with $a_{\max }>1000 \mu \mathrm{m}$. 


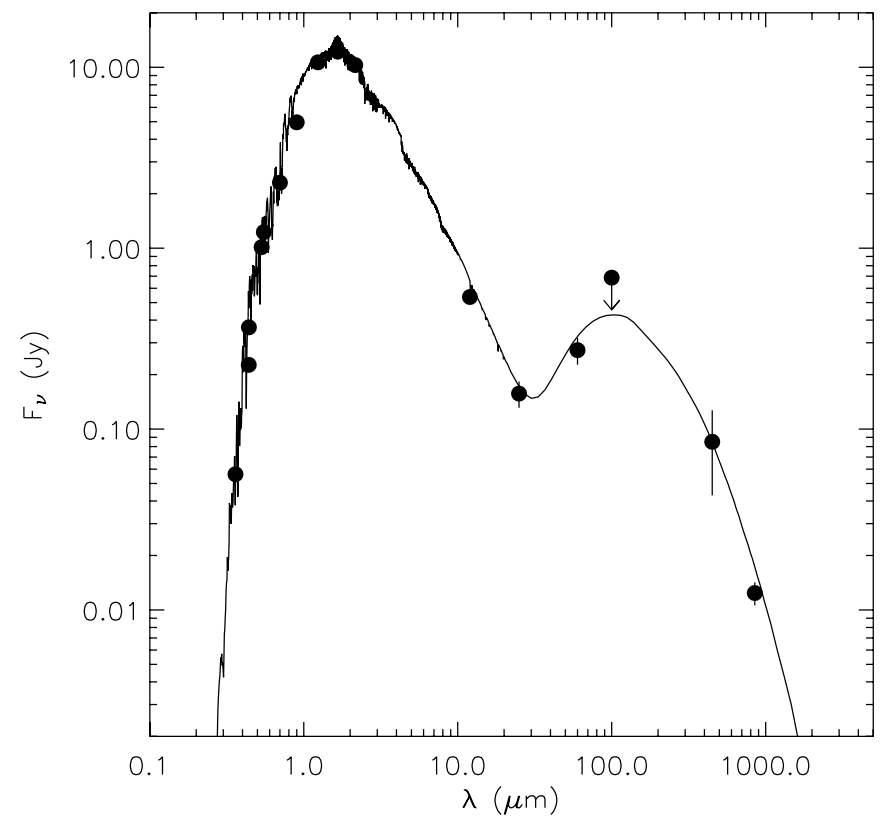

FIG. 7.-SED of AU Mic (data points from Liu et al. 2004 and references therein). The photosphere is fitted by a $3600 \mathrm{~K}$ NextGen model (Hauschildt et al. 1999), and the circumstellar excess emission is fitted using the MC3D code $(\S 4)$ with model parameters listed in Table 1.

blackbody fit in Liu et al. (2004). A firm upper limit of $r_{\text {in }}<$ 17 AU can be set based on the fact that we do not observe a decrease in the intensity of the scattered light with decreasing separation down to 17 AU (Fig. 4; see also Fig. 2 in Liu 2004).

Finally, although we had fixed the value of the power-law index $\gamma$, a brief discussion of its variation is warranted given the change in the SBP with radius. The action of $\gamma$ on the SED is degenerate with the dust size and with the radius of the inner gap. Larger values of $\gamma$ are degenerate with smaller particles and smaller inner gap radii (Fig. 6). Given that at $\leq 33$ AU the power-law index of the SBP decreases to -1.2 (i.e., $\gamma \approx 0.2$ ) and that most of the mid-IR flux is produced close (10-20 AU) to AU Mic, the inner-disk clearing may be smaller than $10 \mathrm{AU}$ in radius. Indeed, recent $H S T$ scattered-light imaging (Krist et al. 2004) detects the disk in to $7.5 \mathrm{AU}$ (although the authors invoke forward scattering to account for the apparent filling in of the inner gap). Compounding this with evidence for an increasing minimum grain size with decreasing radial separation ( $§ 5.1$, we find that inner gap sizes as small as $r_{\text {in }} \sim 1$ AU cannot be ruled out.

Table 1 lists our preferred model parameters for the AU Mic star-disk system. The optical depth of the model along the disk midplane is $\tau_{\|} \approx 0.08$ at both $R$ and $H$ bands, in agreement with the estimates in $\S 3.2$. The model SBP and SED are overplotted on the data in Figures 4 and 7, respectively. Figure $3 c$ shows the noiseless scattered-light model of the AU Mic disk with the same gray scale and contour spacing as the image in Figure $3 a$. The model disk extends to larger radial separations than the AU Mic disk: an effect of the steeper power law of the AU Mic SBP at $>33$ AU.

\section{DISCUSSION}

Two important new results are evident from our simultaneous modeling of the SBP and SED of the AU Mic debris disk: (1) there is a pronounced lack of small $(<0.3 \mu \mathrm{m})$ grains in the inner disk, and (2) the radius of the inner clearing may be smaller (1-10 AU) than estimated (17 AU) from a simple blackbody fit to the IR excess (Liu et al. 2004). The latter point was already discussed in $\S 4.2$ and, given the shortcomings of our model in reproducing the changing slope of the SBP, will not be belabored further. Here we discuss the derived minimum grain size along with recent evidence for its dependence on disk radius. We then focus on the change in slope of the SBP of the AU Mic debris disk and draw a parallel with the $\beta$ Pic system. We propose that identical dynamical processes in the two debris disks can explain the observed homology.

\subsection{Minimum Grain Size as a Function of Disk Radius}

From the model fits to the color and absolute flux of the scattered light from the AU Mic debris disk, we find that the dust grains are between $a_{\min }=0.5_{-0.2}^{+0.5}$ and $a_{\max }=300_{-200}^{+700} \mu \mathrm{m}$ in size (although the $1 \mathrm{~mm}$ upper limit on the maximum grain size is not robust). In reality, our constraints on the grain parameters are valid only over the 50-60 AU region, where we have information from both the $R-H$ color of the scattered light and the SED. We have very few constraints for the outer disk ( $>60 \mathrm{AU}$ ), which is seen only in $R$-band scattered light and is too cold to be detected in emission at wavelengths $<1 \mathrm{~mm}$.

Shortly before receiving the referee report for this paper, sensitive high-resolution $0.4-0.8 \mu \mathrm{m}$ images of AU Mic became available from HST (Krist et al. 2004). These show the debris disk over 7.5-150 AU separations from the star and thus provide complete overlap with our AO data. A brief discussion of the two data sets in the context of the minimum grain size is therefore warranted. For consistency with Krist et al., we redid our surface photometry with the $0.25 \times 0$ ". 25 apertures used by these authors. Because the two data sets have similar angular resolution ( 0 ".04 vs. 0.07$)$, and because the aperture size is much larger than the FWHM of the PSFs, the systematics of the relative photometry should be negligible. The Krist et al. HST F606W $(0.59 \mu \mathrm{m})$ data are consistent with the Kalas et al. (2004) ground-based $R$-band $(0.65 \mu \mathrm{m})$ observations over 50-60 AU from the star, and thus our conclusions about the grain sizes in this region remain unchanged. However, a comparison of the $H$-band and the F606W-band SBP over the region of overlap shows that the F606W $-H$ color changes from $3.0 \pm 0.2 \mathrm{mag}$ (i.e., approximately neutral, since $R-H=2.9$ for AU Mic) at $17-20 \mathrm{AU}$ to $2.0 \pm 0.3 \mathrm{mag}$ at $50-60 \mathrm{AU}$. That is, the debris disk becomes bluer at larger radii. The effect is gradual and is also reported in Krist et al., where it is observed over a narrower wavelength range $(0.4-0.8 \mu \mathrm{m})$ at $30-60 \mathrm{AU}$ from the star.

The neutral color of the dust at $20 \mathrm{AU}$ indicates that the majority of scatterers there are larger than $1.6 \mu \mathrm{m}$. Compared with the minimum grain size $\left(0.5_{-0.2}^{+0.5} \mu \mathrm{m}\right)$ that we derived at 50-60 AU, this indicates that at smaller separations grains are bigger. Such a dependence of grain size on radius would further imply that the radius at which the SBP changes power-law index should be wavelength-dependent, occurring farther away from the star at shorter wavelengths. Evidence for this may indeed be inferred from a comparison between the HST and Keck AO data: in the F606W ACS filter the break in the SBP is seen at $\approx 43 \mathrm{AU}$ (Krist et al. 2004), whereas at $H$ band it occurs near 33-35 AU ( $\S 3.1$; Liu 2004). The indication that the minimum grain size decreases with disk radius is thus confirmed from two independent observations.

Particles smaller than $a_{\text {min }}$ may be removed as a result of either coagulation into larger particles (grain growth), destruction by P-R and/or corpuscular drag, or radiation pressure blowout. Given that $a_{\mathrm{min}}$ is larger than the radiation pressure blowout size $(0.14 \mu \mathrm{m}$, for a radiation pressure to gravity ratio $\beta=0.5$ 
and a grain density of $2.5 \mathrm{~g} \mathrm{~cm}^{-3}$ ), grain collisions and drag forces dominate the dynamics of $>0.14 \mu \mathrm{m}$ grains around AU Mic. Therefore, the origin of the submicron grains scattering visible light at wide separations (where the collision and P-R timescales are longer than the age of the star) may also be primordial: rather than being blown out from the inner disk, these grains may be remnant from the protostellar cloud and never coagulated beyond an ISM grain size distribution.

\subsection{The Change in the SBP Power-Law Index: $A$ Comparison with $\beta$ Pic}

It is not surprising that our preferred model cannot reproduce the detailed structure of our high angular resolution IR image. The model parameters were found only after a coarse sampling of the parameter space and through a number of simplistic assumptions that merely approximate the physical conditions in the AU Mic debris disk. In particular, under the assumption of a uniform grain size and density distribution over 10$1000 \mathrm{AU}$, the MC3D model cannot mimic the SBP slope change at 233 AU and the clumpy substructure over 17-60 AU described in Liu (2004) and confirmed in $\S 3.1$. While the dust clumps are high-order perturbations that may require dynamical considerations for proper modeling, the change in the SBP potentially could be explained in the framework of existing dust disk scenarios.

The occurrence of the power-law break at similar radii in the SBPs of both arms of the projected disk suggests that this is a ringlike structure surrounding the star, rather than a discrete feature at one location in the disk. Models involving dynamical interaction with planets have been proposed to explain ringlike structures in circumstellar disks (e.g., Roques et al. 1994; Liou \& Zook 1999; Kenyon et al. 1999; Kenyon \& Bromley 2004), and the clumpy structure of the AU Mic disk does suggest the presence of unseen planets (Liu 2004). However, such models tend to produce discrete rings, as around HR 4796A and HD 141569A, rather than the radially dimming SBP of the AU Mic disk. Similar changes in the power-law index have also been seen in the SBPs of other resolved circumstellar disks: $\beta$ Pic (Artymowicz et al. 1990; Heap et al. 2000), TW Hya (Krist et al. 2000; Weinberger et al. 2002), and HD 100546 (Pantin et al. 2000; Augereau et al. 2001). A different mechanism, not necessarily involving planets, may be at play in these systems.

The TW Hya and HD 100546 circumstellar disks are gas-rich and have large midplane optical depths, and hence are very much unlike the gas-poor (Roberge et al. 2004), optically thin AU Mic debris disk. However, a comparison with $\beta$ Pic is particularly illuminating because of the similar viewing geometries of the two systems and their identical ages. In the remainder of this section we seek a common disk architecture that can self-consistently account for the broken power-law morphology of the SBPs of these two debris disks.

The SBP of $\beta$ Pic exhibits a break at $5^{\prime \prime}-6^{\prime \prime}(100-120 \mathrm{AU})$ from the star (e.g., Golimowski et al. 1993; Heap et al. 2000), with similar values (from -1 to -4 ) of the power-law index on either side of the break as in the SBP of AU Mic. From $K^{\prime}$-band $(0.21 \mu \mathrm{m})$ AO observations resolving the $\beta$ Pic disk over $11^{\prime \prime} 5-$ 6 ", Mouillet et al. (1997) observe the break at a somewhat smaller radius, $4^{\prime \prime}-4$ ".5 (75-85 AU), with a smaller change in the SBP power-law index (from -1 to -3 ). This may be the inner edge of the SBP break observed in the visible, or it may indicate a wavelength dependence of the $\beta$ Pic break radius similar to the one potentially seen in the AU Mic disk ( $§ 5.1)$. However, because the Mouillet et al. $K^{\prime}$-band data do not extend beyond the optical break radius $\left(6^{\prime \prime}\right)$, and because the change in the power-law index observed at $K^{\prime}$ does not span the full range of power-law indices inferred from optical imaging, this data set will not be considered further.

Artymowicz et al. (1989) model the break in the optical SBP of the $\beta$ Pic disk using two different power laws for the number density of dust particles in the disk for radii less than or greater than $100 \mathrm{AU}$, respectively. Backman et al. (1992) consider the possibility of differing grain sizes, in addition. While either model may correctly describe the architecture of the $\beta$ Pic disk, both are purely phenomenological, as they do not model the physics behind the discontinuity in the disk. Based on the apparent homology between these two debris disks, we believe that a plausible two-component model should be able to explain both systems self-consistently. In light of this, several physical scenarios from the subsequent literature are considered below. We find that none of them offer a unique explanation and propose separate hypotheses in $\S \S 5.2 .3$ and 5.2.4.

\subsubsection{Ice or Comet Evaporation}

Backman et al. (1992) and Pantin et al. (1997) suggest that the discontinuity in the SBP of $\beta$ Pic may correspond to the location of the "ice boundary" in the disk: all dust particles at separations $>100$ AU are covered with ice, while at shorter separations some may not be. This results in a deficiency of highly reflective particles in the inner regions, creating a shallower power-law index for the scattered-light profile. In an optically thin disk the radius of the ice boundary should scale as the square root of the stellar luminosity. Adopting $L_{\mathrm{AU} \text { Mic }}=0.13 L_{\odot}$ (Table 1) and $L_{\beta \text { Pic }}=8.7 L_{\odot}$ and assuming identical grain chemistry in the two systems, we find that the corresponding boundary around AU Mic should scale down to a radius of 13$15 \mathrm{AU}$; this is too close to account for the break at $33 \mathrm{AU}$.

An alternative hypothesis involving dust extraction through evaporation of gas from a reservoir of cometary bodies around $\beta$ Pic is proposed by Lecavelier des Etangs et al. (1996). However, the radial distance of this evaporating reservoir should scale in the same manner as that of the ice boundary. Hence, neither of these two hypotheses can be applied simultaneously to AU Mic and $\beta$ Pic.

\subsubsection{A Belt of Parent Bodies}

A reservoir of parent bodies at a discrete range of separations from AU Mic could explain the kink in the SBP. Gor'kavyi et al. (1997) calculate that the main asteroid belt in the solar system should produce a break in the power-law index of the number density distribution of interplanetary grains from -1.3 to -6.4 at 0.5-3.0 AU from the Sun. These predictions are consistent with empirical data from radar meteors and from impact detectors on spacecraft (Divine 1993). The inner edge of this belt of asteroids in the AU Mic and $\beta$ Pic systems would be at the location of the kinks, at $\sim 33$ and $\sim 110$ AU from the stars, respectively. By continuing the analogy with the solar system, such belts of parent bodies would likely need to be maintained in a discrete range of orbits through mean motion resonances with planets (e.g., Liou \& Zook 1997 and references therein). That is, this particular scenario may provide further indication for the existence of planets in the two disks, in addition to the evidence arising from the their clumpy structure. However, this model is poorly constrained, as we are free to invoke a belt of parent bodies at any distance from either star.

\subsubsection{Collisional Evolution}

For two stars of the same age, the disk around the more massive star is expected to be collisionally evolved out to a 
greater radius because of the inverse scaling of the orbital period (and hence collision frequency) with orbital radius and stellar mass. The collisional timescale for particles of mean size $a$ on a circular orbit of radius $r$ from a star of mass $M_{*}$ is

$$
\begin{aligned}
t_{\mathrm{coll}} & \sim \frac{1}{2 \pi a^{2} n(r) v_{\mathrm{rms}}} \sim \frac{1}{2 \pi a^{2} n_{0} r^{-\gamma}} \frac{r}{h v_{\mathrm{orb}}} \\
& \propto \frac{r}{h} \frac{r^{\gamma+1 / 2}}{M_{*}^{1 / 2} n_{0}}
\end{aligned}
$$

where $v_{\text {rms }}$ is the rms velocity of dust particles, $v_{\text {orb }}$ is their orbital velocity, $h$ is the disk scale height, and $n_{0}$ is the normalization constant for the number density distribution, which we presume scales as $M_{\text {dust }}$. The approximate ratio of the stellar masses of AU Mic and $\beta$ Pic is 0.28 (assuming $M_{\beta \text { Pic }}=$ $\left.1.8 M_{\odot}\right)$ and that of their circumstellar dust masses is $0.2 \pm 0.1$ (where the mass of the $\beta$ Pic disk was taken as the average of the estimates from Sheret et al. 2004 and Dent et al. 2000). Assuming 33 and 110 AU as the radii of the kinks in the corresponding SBPs and that the aspect ratios $r / h$ of the two disks are the same, we find that for $\gamma=1.5_{-0.4}^{+0.3}$ the collisional timescales at the respective separations around the two stars are equal. This would imply SBPs decreasing approximately as $r^{-\gamma-1}=r^{-2.5}$, which is within the ranges found in $\S 3.1$ for AU Mic and in Heap et al. (2000) for $\beta$ Pic and agrees with the fit to the mean AU Mic $H$-band SBP (power-law index of $-2.3 \pm 0.2$; Fig. 4). Therefore, in this scenario the two disk systems scale correctly within the errors, indicating that whatever process we are observing may scale with the mean time between interparticle collisions. Although the collisional timescale at the location of the break in the AU Mic SBP is considerably shorter ( $\sim 1 \mathrm{Myr})$ than the age of the star (8-20 Myr), other, slower processes in the disk, e.g., grain growth, may scale linearly with the time between particle collisions. As noted in $\S 5.1$, grain growth could also explain the observed dependence of grain size with orbital radius.

\subsubsection{Poynting-Robertson Drag}

The observed change in the power-law index of the SBP may be a reflection of the finite lifetimes of submicron grains in the inner disk. Having ruled out radiation pressure as a dominant force on grains larger than $0.14 \mu \mathrm{m}(\S 5.1)$, we propose a hypothesis based on drag forces for grain removal, in particular P-R drag. Although corpuscular drag may dominate the dynamics of dust around M stars (e.g., Fleming et al. 1995), the strength of stellar winds from $\mathrm{M}$ dwarfs remains largely unknown. We therefore ignore corpuscular drag in the following analysis (although see Plavchan et al. 2005 for a discussion of the role of corpuscular drag in the AU Mic disk) and consider only P-R drag. As long as the magnitude of the corpuscular drag force around $\mathrm{AU}$ Mic is not much greater than the magnitude of the P-R drag force, the conclusions remain unchanged.

If $\mathrm{P}-\mathrm{R}$ drag was responsible for the depletion of micron-sized grains in the inner disks of AU Mic and $\beta$ Pic, then the P-R lifetime ( $\left.t_{\mathrm{PR}}\right)$ of the smallest grains $a_{\mathrm{min}}$ should be constant as a function of disk radius $r$ and should equal the age of the stars $\left(t_{\text {age }}\right)$. Note, however, that the size of the smallest grains, $a_{\min }$, is not a constant, but varies under P-R drag as $a_{\min } \propto r^{-2}$. The expression for the P-R lifetime of a particle of size $a$ (see, e.g., Burns et al. 1979),

$$
t_{\mathrm{PR}}(a, r)=\left(\frac{4 \pi a \rho}{3}\right)\left(\frac{c^{2} r^{2}}{L_{*}}\right),
$$

where $\rho$ is the mean grain density $\left(2.5 \mathrm{~g} \mathrm{~cm}^{-3}\right.$ for silicates $)$ and $c$ is the speed of light. Based on our assumption, $t_{\mathrm{PR}}\left(a_{\mathrm{min}}, r\right)=$ $t_{\text {age }}=$ const.

For AU Mic we found $a_{\min } \geq 0.3 \mu \mathrm{m}$ at 50-60 AU and $a_{\min } \geq$ $1.6 \mu \mathrm{m}$ at $17-20 \mathrm{AU}(\S 5.1)$. We obtain $t_{\mathrm{PR}}(0.3 \mu \mathrm{m}, 50 \mathrm{AU})=$ 9.9 Myr and $t_{\mathrm{PR}}(1.6 \mu \mathrm{m}, 20 \mathrm{AU})=8.4 \mathrm{Myr}$. We do not have information about the change in $a_{\mathrm{min}}$ as a function of radius in $\beta \mathrm{Pic}$. We only note that from mid-IR and visual images, Artymowicz et al. (1989) find that they require "few-micron-sized" silicate grains $^{2}$ to model the scattered light at $>6$."0 (>115 AU) from the star. Assuming a minimum grain size of $3 \mu \mathrm{m}$, we find $t_{\mathrm{PR}}(3 \mu \mathrm{m}, 115 \mathrm{AU})=7.8 \mathrm{Myr}$ around $\beta$ Pic. Given the uncertainty in $a_{\mathrm{min}}$, the obtained P-R timescales are not constrained to better than a factor of 1.5-2. Nevertheless, they are remarkably similar and agree well with the ages of AU Mic and $\beta$ Pic.

\subsubsection{Summary of Proposed Scenarios}

We find that dynamical scenarios based on collisional evolution or P-R drag in debris disks offer simpler and more selfconsistent accounts of the homology between the SBPs of the $\mathrm{AU}$ Mic and $\beta$ Pic debris disks, compared to scenarios relying on ice/grain evaporation or belts of orbiting parent bodies. Moreover, both our hypotheses can account for the inferred decrease in the minimum grain size with increasing separation from AU Mic. We therefore conclude that both are plausible. Given the similarities in their predictions, we do not single out which one of them is more likely, but defer that analysis to a more detailed theoretical work. Regardless of which of the two processes is found to be dominant, we can confidently claim that optically thin circumstellar disks exhibiting breaks in their SBPs are observed in transition between a primordial and a debris state.

\section{CONCLUSION}

We have used AO $H$-band observations of scattered light to probe the morphology of the debris disk at 17-60 AU from the young nearby M dwarf AU Mic. We find that the disk is within $\sim 1^{\circ}$ of edge-on and that it exhibits a number of morphological peculiarities: radial asymmetry, spatially resolved clumps, and a change in the power-law index of the surface brightness profile near $33 \mathrm{AU}$. The observed morphology agrees with that reported in Liu (2004) and is suggestive of the existence of planetary perturbers in the disk. No planets are detected down to $1 M_{\mathrm{J}}$ at $>20 \mathrm{AU}$.

We use a Monte Carlo three-dimensional dust disk model to constrain the overall disk parameters by optimizing them against the AU Mic SED and near-IR and optical scattered-light images of the disk (this paper; Kalas et al. 2004). The combined use of SED data tracing the thermal emission from large grains, and of imaging data tracing grain properties, allows us to break several important degeneracies in models of circumstellar disks that cannot be resolved using only one of the two data sets. From the SED we confirm the previously inferred (Liu et al. 2004) circumstellar dust mass of $0.011 M_{\oplus}$, and from the properties of the scattered light we infer that the debris particles are $\geq 0.5_{-0.2}^{+0.5} \mu \mathrm{m}$ in size at 50-60 AU from AU Mic. We find tentative evidence for a maximum grain size of $300_{-200}^{+700} \mu \mathrm{m}$. However, since the data are not sensitive to particles $\gg 1 \mathrm{~mm}$, the result is consistent with no upper limit on the grain size.

\footnotetext{
${ }^{2}$ A second solution involving $1-15 \mu \mathrm{m}$ ice grains is found to be equally plausible. However, its grain properties differ widely from the ones adopted for the AU Mic circumstellar dust in this paper.
} 
Assuming a single dust size and density distribution, we estimate that the radius of the inner-disk clearing is $10 \mathrm{AU}$. However, smaller $(\sim 1 \mathrm{AU})$ gap sizes cannot be ruled out if a shallower density profile (as observed inward of $33 \mathrm{AU}$ ) and/or larger grains in the inner disk (as evidenced from the optical near-IR color of the disk) are adopted. We attribute the lack of submicron particles in the inner disk to either grain growth, destruction by P-R and/or corpuscular drag (for grains $>0.14 \mu \mathrm{m}$ ), or blowout by radiation pressure (for grains $<0.14 \mu \mathrm{m}$ ). All of these mechanisms can explain the increase in relative density of small grains with increasing radius in the disk.

The MC3D model can account for the overall disk profile and colors to first order, although our one-component model fails to reproduce higher order effects, such as the change in power-law index of the SBP. We have discussed a number of scenarios that may be capable of reproducing such a change in the joint context of the AU Mic and $\beta$ Pic debris disks. We find that models dividing the disk into two separate components, with different grain distribution and/or composition, represent the combined SED and imaging data best. In particular, classes of models that scale with the collision or the P-R timescale are most likely to explain both debris disks self-consistently.

Future high dynamic range imaging observations probing closer to AU Mic (e.g., with nulling interferometry in the midIR) will further narrow down the architecture of its debris disk. Photometric and spectroscopic observations with Spitzer could better constrain the SED of AU Mic and could be used to look for spectroscopic features. These could trace small amounts of dust and gas in the inner disk, even if no continuum excess is seen at $<25 \mu \mathrm{m}$.

We are grateful to Randy Campbell, Paola Amico, and David Le Mignant for their guidance in using Keck AO, Keith Matthews and Dave Thompson for advice on using NIRC2, and our telescope operator, Madeline Reed, at the Keck II telescope. We also thank the anonymous referee for his/her constructive comments, Scott Kenyon for a critical review of the draft manuscript, and Laird Close, Eugene Chiang, Ruth Murray-Clay, and Peter Plavchan for insightful discussions. This publication has made use of data products from the Two Micron All Sky Survey, which is a joint project of the University of Massachusetts and the IPAC/California Institute of Technology, funded by the NASA and the NSF, and of the SIMBAD database, operated at CDS, Strasbourg, France. Finally, the authors wish to extend special thanks to those of Hawaiian ancestry, on whose sacred mountain of Mauna Kea we are privileged to be guests. Without their generous hospitality, none of the observations presented herein would have been possible. J. A. E. acknowledges support from a Michelson Graduate Research Fellowship. S. W. was supported by the German Research Foundation (DFG) through the Emmy Noether grant WO 857/2-1.

\section{REFERENCES}

Artymowicz, P., Burrows, C., \& Paresce, F. 1989, ApJ, 337, 494

Artymowicz, P., Paresce, F., \& Burrows, C. 1990, Adv. Space Res., 10, 81

Augereau, J. C., Lagrange, A. M., Mouillet, D., \& Ménard, F. 2001, A\&A, 365,78

Augereau, J. C., \& Papaloizou, J. C. B. 2004, A\&A, 414, 1153

Aumann, H. H., et al. 1984, ApJ, 278, L23

Backman, D., \& Gillett, F. C. 1987, in Cool Stars, Stellar Systems, and the Sun, ed. J. L. Linsky \& R. E. Stencel (Berlin: Springer), 340

Backman, D. E., \& Paresce, F. 1993, in Protostars and Planets III, ed. E. H. Levy \& J. I. Lunine (Tucson: Univ. Arizona Press), 1253

Backman, D. E., Witteborn, F. C., \& Gillett, F. C. 1992, ApJ, 385, 670

Barrado y Navascués, D., Stauffer, J. R., Song, I., \& Caillault, J.-P. 1999, ApJ, 520, L123

Bohren, C. F., \& Huffman, D. R. 1983, Absorption and Scattering of Light by Small Particles (New York: Wiley)

Briggs, R. E. 1962, AJ, 67, 710

Burns, J. A., Lamy, P. L., \& Soter, S. 1979, Icarus, 40, 1

Burrows, A., et al. 1997, ApJ, 491, 856

Carpenter, J. M., Wolf, S., Schreyer, K., Launhardt, R., \& Henning, T. 2005, AJ, 129, 1049

Cashwell, E. D., \& Everett, C. J. 1959, A Practical Manual on the Monte Carlo Method for Random Walk Problems (New York: Pergamon)

Clampin, M., et al. 2003, AJ, 126, 385

Decin, G., Dominik, C., Waters, L. B. F. M., \& Waelkens, C. 2003, ApJ, 598, 636

Dent, W. R. F., Walker, H. J., Holland, W. S., \& Greaves, J. S. 2000, MNRAS, 314, 702

Divine, N. 1993, J. Geophys. Res., 98, 17029

Draine, B. T., \& Lee, H. M. 1984, ApJ, 285, 89

Draine, B. T., \& Malhotra, S. 1993, ApJ, 414, 632

Fleming, T. A., Schmitt, J. H. M. M., \& Giampapa, M. S. 1995, ApJ, 450, 401

Golimowski, D. A., Durrance, S. T., \& Clampin, M. 1993, ApJ, 411, L41

Gor'kavyi, N. N., Ozernoy, L. M., Mather, J. C., \& Taidakova, T. 1997, ApJ, 488,268

Gorlova, N., et al. 2004, ApJS, 154, 448

Greaves, J. S., et al. 1998, ApJ, 506, L133

Habing, H. J., et al. 2001, A\&A, 365, 545

Hartkopf, W. I., \& Mason, B. D. 2003, Sixth Catalog of Orbits of Visual Binary Stars: Calibration Candidates (Washington, DC: US Naval Obs.), http:// ad.usno.navy.mil/wds/orb6/orb6c.html

Hauschildt, P. H., Allard, F., \& Baron, E. 1999, ApJ, 512, 377

Heap, S. R., Lindler, D. J., Lanz, T. M., Cornett, R. H., Hubeny, I., Maran, S. P., \& Woodgate, B. 2000, ApJ, 539, 435
Holland, W. S., et al. 1998, Nature, 392, 788 2003, ApJ, 582, 1141

Kalas, P., Deltorn, J., \& Larwood, J. 2001, ApJ, 553, 410

Kalas, P., Larwood, J., Smith, B. A., \& Schultz, A. 2000, ApJ, 530, L133

Kalas, P., Liu, M. C., \& Matthews, B. C. 2004, Science, 303, 1990

Keenan, P. C. 1983, Bull. d'Inf. Cent. Donnees Stellaires, 24, 19

Kendall, M., \& Stuart, A. 1977, The Advanced Theory of Statistics, Vol. 1:

Distribution Theory (4th ed.; London: Griffin)

Kenyon, S. J., \& Bromley, B. C. 2002, AJ, 123, 1757 2004, AJ, 127, 513

Kenyon, S. J., Wood, K., Whitney, B. A., \& Wolff, M. J. 1999, ApJ, 524, L119 Koerner, D. W., Sargent, A. I., \& Ostroff, N. A. 2001, ApJ, 560, L181

Krist, J. E., Ardila, D. R., Golimowski, D. A., Clampin, M., Ford, H. C., Illingworth, G. D., Hartig, G. F., \& ACS Science Team. 2004, AAS Meeting, $205,17.12$

Krist, J. E., Stapelfeldt, K. R., Ménard, F., Padgett, D. L., \& Burrows, C. J. 2000, ApJ, 538, 793

Laureijs, R. J., Jourdain de Muizon, M., Leech, K., Siebenmorgen, R., Dominik, C., Habing, H. J., Trams, N., \& Kessler, M. F. 2002, A\&A, 387, 285 Lecavelier des Etangs, A., Vidal-Madjar, A., \& Ferlet, R. 1996, A\&A, 307, 542 Leinert, C., Röser, S., \& Buitrago, J. 1983, A\&A, 118, 345

Liou, J., \& Zook, H. A. 1997, Icarus, 128, 354 1999, AJ, 118, 580

Liu, M. C. 2004, Science, 305, 1442

Liu, M. C., Matthews, B. C., Williams, J. P., \& Kalas, P. G. 2004, ApJ, 608, 526

Mannings, V., \& Barlow, M. J. 1998, ApJ, 497, 330

Mathis, J. S., Rumpl, W., \& Nordsieck, K. H. 1977, ApJ, 217, 425

Meyer, M. R., et al. 2004, ApJS, 154, 422

Moro-Martín, A., \& Malhotra, R. 2002, AJ, 124, 2305

Mouillet, D., Lagrange, A.-M., Beuzit, J.-L., \& Renaud, N. 1997, A\&A, 324,1083

Nakano, T. 1990, ApJ, 355, L43

Ozernoy, L. M., Gorkavyi, N. N., Mather, J. C., \& Taidakova, T. A. 2000, ApJ, 537, L147

Pantin, E., Lagage, P. O., \& Artymowicz, P. 1997, A\&A, 327, 1123

Pantin, E., Waelkens, C., \& Lagage, P. O. 2000, A\&A, 361, L9

Perryman, M. A. C., et al. 1997, A\&A, 323, L49

Plavchan, P., Jura, M., \& Lipscy, S. J. 2005, ApJ, submitted

Pourbaix, D. 2000, A\&AS, 145, 215

Quillen, A. C., \& Thorndike, S. 2002, ApJ, 578, L149

Reach, W. T., et al. 1995, Nature, 374, 521 
Roberge, A., Weinberger, A. J., \& Feldman, P. D. 2004, in Proc. Second TPF/ Darwin Int. Conf., Dust Disks and the Formation, Evolution, and Detection of Habitable Planets, ed. D. Bui (Pasadena: JPL), http://planetquest1.jpl.nasa. gov/TPFDarwinConf/index.cfm

Roques, F., Scholl, H., Sicardy, B., \& Smith, B. A. 1994, Icarus, 108, 37

Schneider, G., et al. 1999, ApJ, 513, L127

Sheret, I., Dent, W. R. F., \& Wyatt, M. C. 2004, MNRAS, 348, 1282

Silverstone, M. D. 2000, Ph.D. thesis, Univ. California, Los Angeles

Song, I., Weinberger, A. J., Becklin, E. E., Zuckerman, B., \& Chen, C. 2002, AJ, 124, 514

Spangler, C., Sargent, A. I., Silverstone, M. D., Becklin, E. E., \& Zuckerman, B. 2001, ApJ, 555, 932

Stern, S. A. 1996, A\&A, 310, 999

Sylvester, R. J., \& Mannings, V. 2000, MNRAS, 313, 73

Takeuchi, T., \& Artymowicz, P. 2001, ApJ, 557, 990
Torres, C. A. O., \& Ferraz Mello, S. 1973, A\&A, 27, 231

Wahhaj, Z., Koerner, D. W., Ressler, M. E., Werner, M. W., Backman, D. E., \& Sargent, A. I. 2003, ApJ, 584, L27

Weinberger, A. J., Becklin, E. E., Schneider, G., Smith, B. A., Lowrance, P. J., Silverstone, M. D., Zuckerman, B., \& Terrile, R. J. 1999, ApJ, 525, L53

Weinberger, A. J., et al. 2002, ApJ, 566, 409

Weingartner, J. C., \& Draine, B. T. 2001, ApJ, 548, 296

Wilner, D. J., Holman, M. J., Kuchner, M. J., \& Ho, P. T. P. 2002, ApJ, 569, L115

Wizinowich, P., et al. 2000, PASP, 112, 315

Wolf, S. 2003, Comput. Phys. Commun., 150, 99

Wolf, S., \& Henning, T. 2000, Comput. Phys. Commun., 132, 166

Wyatt, M. C. 2003, ApJ, 598, 1321

Wyatt, M. C., \& Dent, W. R. F. 2002, MNRAS, 334, 589

Zuckerman, B., Song, I., \& Webb, R. A. 2001, ApJ, 559, 388 